\title{
The Importance of Psychopathological Assessment in Patients with Migraine
}

Irene Muñoz and Angel L Guerrero*

Psychiatry and Neurology Departments, University Hospital, Valladolid, Spain

"Corresponding author: Angel L Guerrero, Psychiatry and Neurology Departments, University Hospital, Valladolid, Spain, E-mail: gueneurol@gmail.com

Received date: April 12, 2015; Accepted date: April 21, 2015; Published date: April 27, 2015

Copyright: @ 2015 Muñoz I. This is an open-access article distributed under the terms of the Creative Commons Attribution License, which permits unrestricted use, distribution, and reproduction in any medium, provided the original author and source are credited.

Keywords: Chronic migraine; Medication overuse; Obsessivecompulsive trait/disorder; Psychological profile; Personality

\section{Commentary}

The psychopathological assessment of patients with migraine is a topic of growing interest. An abnormal psychological profile may have important clinical and therapeutic implications. There are certain personality profiles related to pain coping patterns that seem to complicate the management of migraine. For patients with increased susceptibility to stress, standard preventive treatment of migraine is often unsuccessful. As a result, migraine chronification represents nowadays a difficult treatment challenge.

One of the main risk factors for chronicity of migraine is the overuse of symptomatic medication and some patients with obsessivecompulsive or anancastic personality disorders have a tendency to compulsive medication intake; so, it would be advisable to screen for obsessive-compulsive traits in migraine patients.

Several studies support this reasoning: Curone [1] showed that obsessive-compulsive personality trait is considered a possible factor of refractoriness to preventive treatment in chronic migraine, and that it might also lead to constant medication overuse. He noted that obsessive-compulsive trait in migraine patients is often underdiagnosed, which may significantly boost the risk of developing a chronic migraine and overuse of medication.

Curone and Cupini $[2,3]$ showed that compulsive behaviour could represent a major risk of medication overuse in migraine patients. They explained that subclinical obsessive-compulsive disorder is a psychiatric comorbidity that can alter the clinical course of migraine and affect efficacy of preventive treatment.

One of Curone's most recent studies [4] confirmed the hypothesis that obsessive-compulsive trait is a predictive factor of poor response in patients overusing medication, leading to chronic migraine and comorbid depression. Curone used drugs to treat major depressive disorders like duloxetine, in order to drop the frequency of migraine attacks and to limit the overuse of medication in chronic migraine patients with comorbid depression by decreasing their depression scores. However, he pointed out that patients with obsessivecompulsive disorder coexisting with chronic migraine and depression had similar depression scores and neither reduced the frequency of migraine attacks nor limited the overuse of medication when treated with the same drug.

Finally Luconi [5] stated that psychological factors can affect the response to chronic migraine treatment. These were not also factors related to the severity of migraine, but even fundamental components of the pathology.

In conclusion, psychopathological assessment has a prognostic significance and can provide useful information to allow the clinician to choose between different therapeutic options in migraine patients. Therefore, it should be systematically performed in patients with chronic migraine and medication overuse. Obsessive-compulsive disorders have a prognostic relevance and favor a poor response with persistent disability in these patients.

\section{References}

1. Curone M, D'Amico D, Bussone G (2012) Obsessive-compulsive aspects as predictors of poor response to treatments in patients with chronic migraine and medication overuse. Neurol Sci 33 Suppl 1: S211-213.

2. Curone M, Tullo V, Mea E, Proietti-Cecchini A, Peccarisi C, et al. (2011) Psychopathological profile of patients with chronic migraine and medication overuse: study and findings in 50 cases. Neurol Sci 32 Suppl 1: S177-179.

3. Cupini LM, De Murtas M, Costa C, Mancini M, Eusebi P, et al. (2009) Obsessive-compulsive disorder and migraine with medication-overuse headache. Headache 49: 1005-1013.

4. Curone M, Tullo V, Savino M, Proietti-Cecchini A, Bussone G, et al. (2013) Outcome of patients with chronic migraine with medication overuse and depression after duloxetine: influence of coexisting obsessive compulsive disorder. Neurol Sci 34: S175-S177.

5. Luconi R, Bartolini M, Taffi R, Vignini A, Mazzanti L, et al. (2007) Prognostic significance of personality profiles in patients with chronic migraine. Headache 47: 1118-1124. 